\title{
الإعلام المرئي وتشكيل الوعي في المجتمع المصري \\ * "دراسة تطبيقية في مدينة الفيوم"
}

شيماء محمد محمد أحمد عرفة*

shimaaarafa383@gmail.com

الكلمات المفتاحية:

الإعلام - وسائل الإعلام المرئي - الوعي - المجتمع المصري.

\section{إثكالية الاراسة:}

تحاول هذه الدراسة تتبع أثر انتثار وسائل الإعلام المرئية، ودراسة تأثيرها على وعي الشباب، بهدف الوقوف على النتائج الدترتبة على تعرض الثباب

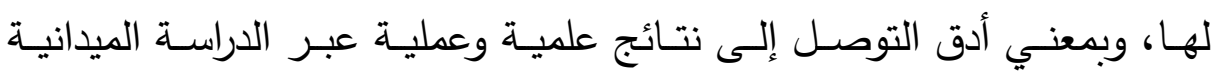
والتحليلية لأهم تأثيرات وسائل الإعلام المرئية على جوانب الحياة الاجتماعية والسياسية والثقافية والصحية والدينية والبيئية لدى الثباب، باعتبارهم الفئة الأكثر

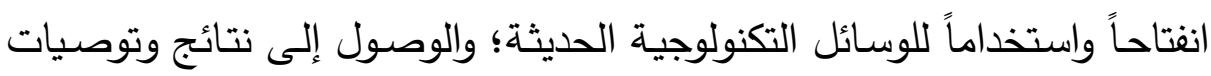

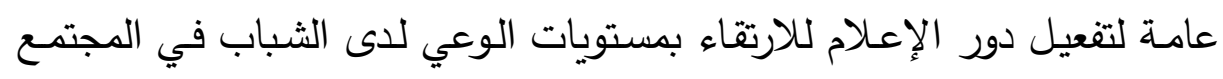

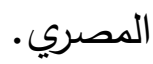

* أُطُروحةٌة ماجِستير في علم الاجتماع الإعلام - كلية الآداب - جامعة الفيوم. * باحثة دكتوراة - كلية الآداب - جامعة الفيوم.

شيماء محمد عرفة

(الإعلام المرئي وتشكيل الوعي في المجتمع المصري....) 


\section{أهمية (لارباسة:}

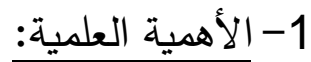

1- إثراء مجال البحث العلمي في فرع من فروع علم الاجتماع وهو علم

$$
\text { الاجتماع الإعلامي. }
$$

2- رصد الدور الذي يلعبه الإعـلام المرئسي في تشكيل وعي الأفراد في

$$
\text { المجتمع المصري. }
$$

3- تحليل الواقع الإعلامي في ضوء التغيرات التي تتتج عنه.

$$
\text { 2- الأهمية التطبيقية: }
$$

1- الإسـهام في تقديم تصـور مقترح لأهم السلبيات الناتجـة عن الإعـلام

$$
\text { المرئي في المجتمع. }
$$

2- التأكيد على أهميـة الوصـول إلى إسـتراتيجية واضـحة لتحقيـق هـدف أساسي للإعلام للوصول الى سياسة إعلامية هادفة تعمل على تنمية الفرد والمجتمع وصقل وعيه تجاه قضاياه الأساسية. 3- تقديم حلول مقترحة لتفعيل دور الإعلام للارتقاء بمستويات الوعي لدى أفراد المجتمع المصري؛ مثل ضرورة تخصيص وحدات أو أقسام داخل مراكز البحوث الاجتماعيـة والتربويـة، تتولي رصد الظواهر الثقافيـة الاجتماعية المستجدة، المصاحبة للتغير الاجتماعي والتقدم التكنولوجي، كالمعلوماتيـة، والانترنـتـ، والهواتـف النقالــة، والهندسـة الوراثيـة.. الــخ، وتثــيص التـأثيرات المتبادلــة بـين تلــك الظــواهر وعناصــر البنــاء 
الاجتمـاعي، وانعكاسـاتها على الأفراد، وتقديم الحلول الفوريـة لمواجهة آثارها، ونشر ثقافة متكاملة الجوانب حيال ترشيد استخدامها.

\section{أهداف الدراسةة:}

\section{تتطلق هذه الاراسة من هدف رئيسي وهو:}

1- التعرف على الدور الذي يلعبـه الإعلام المرئسي في تشكيل الوعي للدى

\section{الشباب في المجتمع المصري.}

\section{ويتفرع من هذا الهدف الرئيسي عدة أهداف فرعيه:}

1- التعرف على دور الإعلام المرئي في تثكيل الوعي الاجتماعي.

2- التعرف على دور الإعلام المرئي في تثكيل الوعي السياسي.

3- التعرف على دور الإعلام المرئي في تثكيل الوعي الثقافي.

4- التعرف على دور الإعلام المرئي في تشكيل الوعي الصحي.

5- التعرف على دور الإعلام المرئي في تثكيل الوعي الديني.

6- التعرف على دور الإعلام المرئي في تشكيل الوعي البيئي.

2- تقديم رؤيسة استشـرافية لتفعيل دور الإعـلام المرئسي للارتقـاء بمسـويات الوعي لاى الثباب في المجتمع المصري.

\section{تساؤلات الاربسة:}

\section{تسعى هذه الدراسة إلى الإجابة عن تساؤل رئيسي:}

1- ما هو الدور الذي يلعبه الإعلام المرئي في تثكيل بعض أثكال الوعي

لأفراد المجتمع المصري؟ 


\section{ومن ثم الإجابة عن تساؤلات فرعية:}

1- كيـف يـؤثر الإعـام المرئي على تشـكيل الـوعي الاجتمـاعي لأفراد

$$
\text { المجتمع المصري؟ }
$$

\section{وذلك من خلال الإجابة على عدة تساؤلات منها:}

- ما هي أهم المشكلات الاجتماعية التي يعاني منها المجتمع؟ - - كيف أثرت وسائل الإعلام المرئية على تعريف أفراد المجتمع المصري

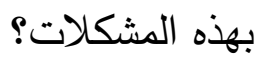

- مـا هي أكثر وسـائل الإعـلام المرئي التي يعتمد عليها أفراد المجتمع - المصري لمتابعة القضايا والمشكلات الاجتماعية؟

2- كيف يؤثر الإعـلام المرئي على تشكيل الوعي السياسـي لأفراد المجتمـع المصري؟

\section{وذلك من خلال الإجابة على عدة تساؤلات منها:}

- ما هي أهم القضايا السياسية التي يهتم أفراد المجتمع المصري بمتابعتها

$$
\text { عبر وسائل الإعلام المرئية؟ }
$$

- - كيف أثرت وسائل الإعلام المرئي على تعريف أفراد المجتمع المصري

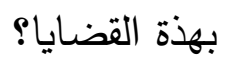

- - مـا هي أكثر وسـائل الإعـلام المرئي التي يعتمد عليها أفراد المجتمع المصري في التعرف على القضايا والأحداث السياسية الجارية؟ 
3- كيف يـؤثر الإعـلام المرئي على تثـكيل الـوعي التثـافي لأفراد المجتمـع

$$
\text { المصري؟ }
$$

\section{وذلك من خلال الإجابة على عدة تساؤلات منها:}

- - ما هي أهم القضايا الثقافية التي يهتم أفراد المتمع المصري بمتابعتها

$$
\text { عبر وسائل الإعلام المرئية؟ }
$$

- - مـن أين يستقي أفراد المجتمـع المصـري معلومـاتهم وآرائهم حول هذه القضايا الثقافية؟

4- كيف يؤثر الإعـلام المرئي على تشكيل الوعي الصـحي لأفراد المجتمـع

$$
\text { المصري؟ }
$$

\section{وذلك من خلال الإجابة على عدة تساؤلات منها:}

- مـا هـي أهـم الموضـوعات الصــية التي يهتم أفراد المجتمع المصـري بمتابعتها عبر وسائل الإعلام المرئية؟

- - هـل تلعـب البـرامج الصـحية دور في تثـكيل الثقافـة الصـحية لأفـراد

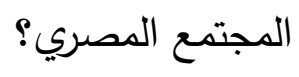

5- كيف يـؤثر الإعـلام المرئي على تشـيل الـوعي الـديني لأفـراد المجتمـع

$$
\text { المصري؟ }
$$

\section{وذلك من خلال الإجابة على عدة تساؤلات منها:}

- مـا هـي أهم المصـادر التي يعتمـد عليهـا أفراد المجتمـع المصـري في المي

الحصول على المعلومات الدينية؟

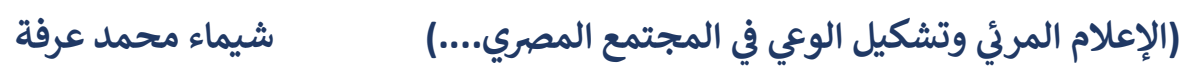


- - هل تقوم البرامج الدينية المقدمـة عبر وسـائل الإعلام المرئي بدور في تشكيل الثقافة الدينية لاى أفراد المجتمع المصري؟

6- كيـف يـؤثر الإعـلام المرئي على تشـكيل الـوعي البيأي لأفـراد المجتــع المصري؟

\section{وذلك من خلال الإجابة على عدة تساؤلات منها:}

- - مـا هـي أهـم أهـم القضــايا البيئيـة التي يهتم أفـراد المجتمـع المصـري

بمتابعتها عبر وسائل الإعلام المرئية؟

- ما هو الدور الذي تلعبه وسائل الإعلام المرئية في تعريف أفراد المجتمع المصري بالمشكلات البيئية وكيفية معالجتها؟

- ما هي العقبات التي تحول دون تعريف أفراد المجتمع المصري بالقضايا

$$
\text { والمشكلات البيئية؟ }
$$

2-كيف يمكن تفعيل دور وسـائل الإعلام المرئي للارتقاء بالوعي لدى أفراد

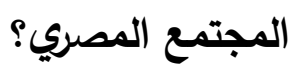

ويتفرع من هذا التساؤل الرئيسي عدة تساؤلات فرعيه:

1- كيـف يمكـن تفعيـل دور وسـائل الإعـلام المرئـي للارتقــاء بـالوعي

الاجتماعي لأفراد المجتمع المصري؟

2- كيف يمكن تفعيل دور وسائل الإعلام المرئي للارتقاء بالوعي السياسي

لأفراد المجتمع المصري؟

3- كيف يمكن تفعيل دور وسائل الإعلام المرئي للارتقاء بالوعي الثقافي

لأفراد المجتمع المصري؟

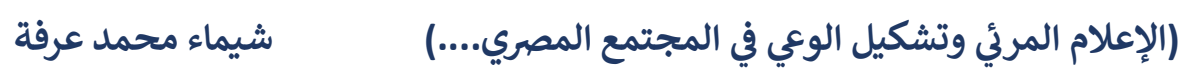


4- كيف يمكن تفعيل دور وسائل الإعلام المرئي للارتقاء بالوعي الصحي لأفراد المجتمع المصري؟ 5- كيف يمكن تفعيل دور وسـائل الإعـلام المرئي للارتقاء بالوعي الديني لأفراد المجتمع المصري؟ 6- كيف يمكن تفعيل دور وسـائل الإعـلام المرئي للارتقاء بالوعي البيئي لأفراد المجتمع المصري؟

\section{نوع الدراسة:}

Descriptive تنتــي هـذه الدراسـة إلـى نمــ الدراســات الوصــفية والتي تستهدف دراسة الحقائق الراهنة المتعلقة بظاهرة أو موقف Research أو مجموعة من الأفراد، وتستهدف تقرير خصائص ظاهرة معينة أو موقف معين يغلب عليـة صـفة التحديـد، وتعتمـــ على جــع الحقـائق وتحليلهـا وتفــيرها لاستخلاص دلالاتها، ومن ثم تمكننا من إصدار تعميمات بشأن الظاهرة التي نقوم بدراستها، وتهدف هذه الدراسـة إلى وصف وتحليـل دور الإعـلام المرئي بوسائله المختلفة (التليفزيون - الفضـائيات - الإنترنت - الهاتف المحمول) في تشكيل بعض أثكال الوعي (الاجتمـاعي - السياسي - الثقافي - الصـحي الإنـي الديني - البيئي) لدى الثباب في مدينة الفيوم؛ وذلك لاستخلاص النتائج في ضوء تساؤلات وأهداف الدراسة. 


\section{منهج الاراسة:}

\section{1- منهج المسح الاجتماعي:}

اعتمدت هذه الدراسة على منهج المسح الاجتماعي بالعينة حيث أن مجتمع

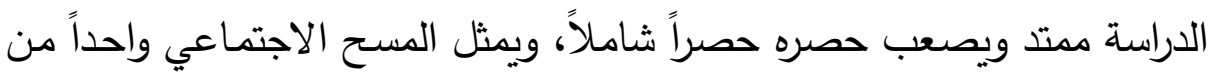
أثهر طرق البحث الاجتماعي الكمي ويقصد به "عملية جمع البيانات بطريقة

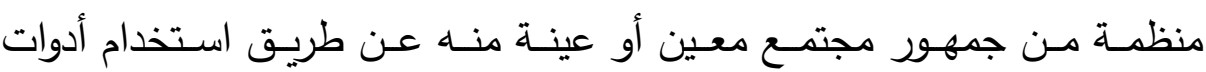
الاستبيان والمقابلة وغيرها، ويجري المسح الاجتماعي للحصول على المعلومات الإحصائية حول قضية معينة أو مشكلة تحتاج إلى حل أو لاختبار فعالية نظرية موجودة، وهذا يشمل على قياس ظواهر مختلفة واستخلاص نتائج حولها لتأكيد نمط من علاقة السبب والنتيجة، وتتمثل أهمية الدسح الاجتماعي في اعتباره أحد الطرق التي يفضل استخدامها في البحوث الوصفية، تلك التي تهدف إلى تقديم

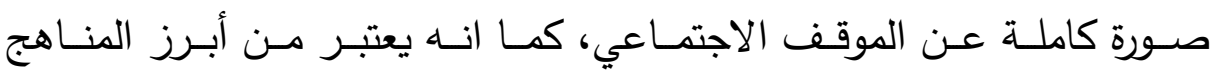
المستخدمة في مجال الدراسات الإعلامية.

وقد تم استخدام منهج الدسح الاجتماعي على عينة عشوائية من شباب مدينة الفيوم قوامها (150) مفردة من سن 18 - 35 سنة من الذكور والإناث، وذلك لأن مجتمع البحث يصعب حصره حصراً شاملاً؛ بهدف التعرف على مدى تأثير وسائل الإعلام المرئية في تثكيل وعي الثباب في الهجتمع المصري. وتم اختيار فئة الثباب لأن هذه المرحلة هي التي يتثكل خلالها الوعي؛ بالإضافة الإنة

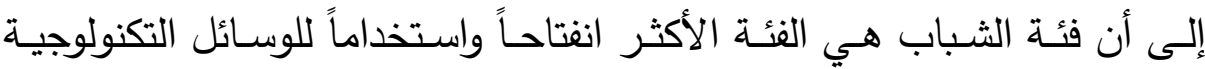

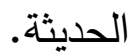

شيماء محمد عرفة

(الإعلام المرئي وتشكيل الوعي في المجتمع المصري....) 


\section{2-منهج تحليل المضمون:}

وهو عملية تحليل منظم لأي رسالة اتصالية أياً كان نوعها، كما يعرف بأنه إسلوب للبحث يهدف إلى الوصف الموضوعي المنظم والكمي للمحتوى الظاهر للاتصال (ويقصد بالاتصـال كل المعاني التي يعبر عنها بالرمز أو الكلمة أو الصـوت أو الصـورة) ويهدف إلى الإجابة عن تساؤلات مثل: من الذي يقول لئل وماذا ولمن وكيف وما هي الآثار المترتبة على ذلك؟ ، ويشتمل على وصف

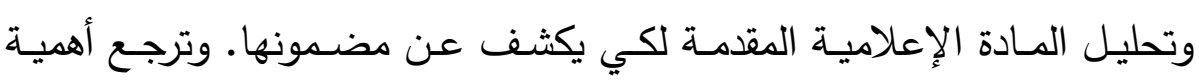
تحليل المضمون إلى تحقيق ثلاثة أهداف رئيسية وهي: 1- تقديم مفاتيح أو علامات حول حقيقة الأهداف والسياسـات التي تسعى إليها النظم الاجتماعية والقيم السائدة.

2- يبحث في طبيعة الرسائل الإعلامية باعتبارها نظامـاً مُعبراً عن حقيقة التأثير المتبادل بين الأفراد. 3- يقدم لنا أساساً لإظهار ما يسببه الفعل من نتائج يمكن مُلاحظتها.

واعتمدت الدراسة الحالية على منهج تحليل المضمون بهدف تحليل محتوى المـادة الإعلاميـة المقدمة في عينـة من البرامج الاجتماعيـة والثقافيـة والسياسية والدينية والصحية المقدمة عبر الفضائيات المصرية والتي تم اختيارها من قبل عينة الدراسة بهدف التعرف على مضمون هذه البرامج للكثف عن الدور الذي تلعبـه في تشكيل وعي الجمهور حول القضـايا المختلفـة التي تتناولهـا. وهذه البرامج هي أولاً: البرنامج الاجتماعي والثقافي "الستات مايعرفوش يكدبوا"، ثانياً: 
البرنامج السياسي "كل يوم"، ثالثًاً: البرنامج الصحي "الدكتور"، لربعاً: البرنامج الديني "والله أعلم"؛ وتم اختيار هذه البرامج بنـاء على استطلاع رأي تم طرحهـ على عينة الدراسة لاختيار البرامج الأكثر مشاهدة في كل محور ، حيث تعتبر هذه البرامج الأكثر مشاهدة من قبل عينة الدراسة وبالتالي الأكثر تأثيراً في عملية تثكيل الوعي؛ بالإضافة إلى تنوع مضامينها الإعلامية تبعاً لأهداف الدراسة.

\section{أدوات الادراسية:}

\section{:Questionnaire الاستبيان -1}

بما أنه المنهج المستخدم هو منهج المسح الاجتماعي فإن الأداة التي تم استخدامها هي آداة الاستبيان، حيث تم تصميم استمارة استبيان للتعرف على مدى تأثير وسائل الإعلام المرئية في تثكيل وعي الثباب طبقاً لأهداف الدراسة وتساؤلاتها وتم تطبيقها على عينة الدراسة.

\section{2- استمارة تحليل المضمون:}

كذلك تم الاستعانة باستمارة تحليل المضمون كآداة لجمع البيانات الخاصـة بتحليل مضمون المادة الإعلامية المقدمة في البرامج التي تم اختيارها، فهو آداة تستخدم لوصف المحتوى الظـاهر والمضـمون الصـريح للمـادة الإعلاميـة المـراد تحليلها مـن حيث الشكل والمضـمون؛ وتهدف استمارة تحليل المضـمون إلى الكثف عن مضامين المادة الإعلامية المقدمة في البرامج المختارة _ والتي بلغ قوامهـا أربعـة بـرامج بإجمـالي عـدد حلقـات 80 حلقـة_ـــن حيـث المضـمون والأسلوب والأهداف والثكل واللغة.

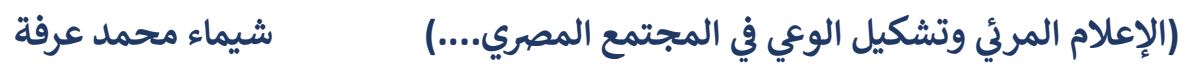




\section{نتائج الاراسة:}

توصلت الدراسة الراهنة إلى أن الإعلام المرئي يلعب دوراً هاماً في تشكيل وعي الأفراد في المجتمع المصري، ويعتبر الإنترنت أكثر وسائل الإعلام المرئي انتشاراً واستخداماً بين جيل الثباب حيث احتل المرتبة الأولى بنسبة 92 \%؛ وبالتالي فهو أكثر تأثيراً على تثكيل آرائهم واتجاهاتهم ووعيهم. كما أكدت الدراسة على تدني مستويات الوعي لدى الثباب خاصـة الوعي الثقافي والبيئي على الرغم من الثورة المعلوماتية الراهنة والتقدم التكنولوجي في مجال الاتصال وتكنولوجيا المعلومات.

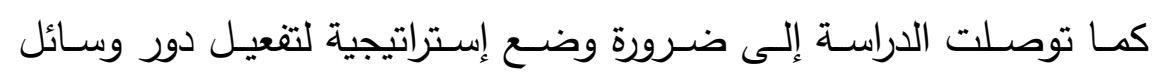
الإعلام المختلفة للارتقاء بمستويات الوعي لأفراد المجتمع المصري.

\section{توصيات الاراسة:}

1- ضـرورة العمل على وضـع إسـتراتيجية إعلاميـة جديـدة واضـحة المـنهج والمُحتوى؛ تهدف في المقام الأول إلى بناء الفكر وتعزيز الوعي من خلال إنتاج منظومـة صناعة إعلامية محترفة في كافة التخصصـات الاجتماعية والسياسية والثقافية والاقتصادية والبيئية والصحية والأمنية.....؛؛ وذلك لما تمتلكـهـ هذه الوسـائل مـن أهميـة وفاعليـة في النهوض بالمستوى الفكري Soft Power والثقافي والحضـاري لأفراد المجتمع باعتبارها القوة الناعمة التي يمكنها أن تؤثر على قناعـات ومبادئ الجمهور مـن خـلال وسـائط الاتصال المختلفة التي تمتلكها خاصة في ظل التطور التكنولوجي وظهور 
الإعلام الرقمي Digital Media الذي أصبح يمثل تطوراً كبيراً ومتفوقاً على وسائل الإعلام التقليدية الأخرى خاصة بين جيل الثباب.

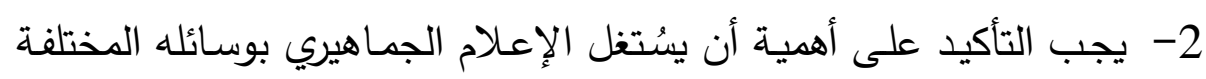

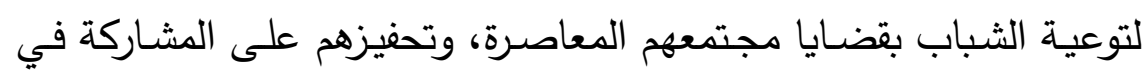

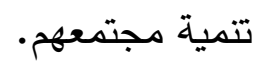

3- كذلك توصي الدراسة بأهمية إقامة خطط وإستراتيجيات تعاونية بين الهيئات

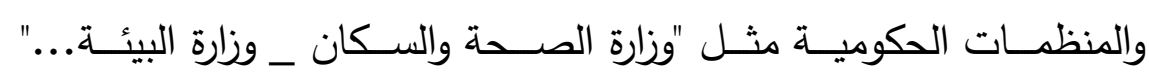

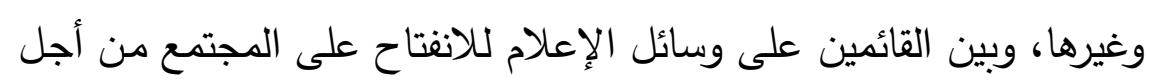
تحسين وعي أبناءه بالقضايا الصحية والبيئية والأمنية وغيرها.

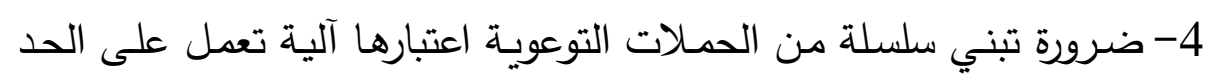
من انتثار السلوكيات السلبية من خلال عرضها بإسلوب بسيط يمكن من فن لئل

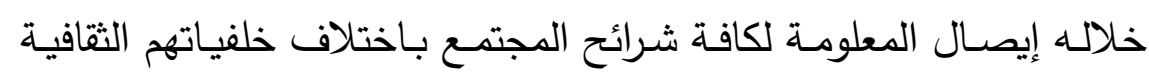
والتعليمية والاجتماعية، وتعتبر وسائل الإعلام المرئي منصات لتحقيق هذه بأهئ

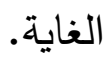




\section{Visual Media and Awareness Formulation in the Egyptian Society "An Applied study in Fayoum City' $\underline{\text { Shimaa mohammed mohammed arafa }}$}

This study seeks to trace the effect of means of visual media, and its effect on people`s awareness. It aims to find out the results of getting detached to these means of media, In other words, reaching scientific and effective results through objective and field study for measuring the effect of mass media on people's "Social - Political - Cultural Health - Religious -Environmental" awareness, and reaching general results and recommendations about the role of media to increase people`s awareness effectively.

The study aims to identify some types of awareness in the Egyptian society and the role of visual media in forming this awareness, through revealing the role of different means of visual media such as 'Television - Satellite - Internet Social Media - Smart Phone" in forming different types of awareness such as "Social - Political - Cultural - Health Religious - Environmental" awareness of Egyptian youth. This study although aims to present a future vision to make the role of visual media effective in increasing the Egyptians awareness.

شيماء محمد عرفة

(الإعلام المرئي وتشكيل الوعي في المجتمع المصري....) - (الم) 


\section{Methodology:}

This study is a descriptive one that aims to study the relation between means of visual media and people's awareness. This study is conducted through survey method using questionnaire tool on a random sample of 150 person of both gender from 18-35 years old. Content analysis is also used to analyze Media content presented in the programs which are the sample of study.

\section{Results:}

- The study finds out that visual media has an important role in formulating awareness in the Egyptian society. Internet is the most popular mean of media among youth as it comes in the first place by $92 \%$, so it has more effect on forming their opinions, approaches, and awareness.

- The study also shows the decline of youth awareness particularly the cultural and environmental awareness, despite of the information revolution and technological progress in communication field.

- The study also shows the importance of making a strategy to improve the role of mass media effectively to increase the Egyptian awareness. 